\title{
The management of diagnosed heart failure in older people in primary care
}

\author{
Nicholas R Jones, FD Richard Hobbs, Clare J Taylor \\ Nuffield Department of Primary Care Health Sciences, University of Oxford, Oxford OX2 6GG \\ Correspondence: Dr Clare J Taylor clare.taylor@phc.ox.ac.uk
}

\begin{abstract}
Heart Failure (HF) is a common condition affecting predominantly older people. Symptoms include breathlessness and fatigue which can significantly impact on quality of life. HF rarely occurs in isolation with most patients having several co-existing diseases and requiring multiple medications. There is a large evidence base for treatment of HF with reduced ejection fraction, or HFrEF, however many of the trials did not include older people with multimorbidity so their findings should be applied to this group with some caution. The evidence for treatment of HF with preserved ejection fraction, or HFpEF, is much less well established in all age groups.
\end{abstract}

Older people with HF are usually managed in primary care with input from specialist HF teams when needed. General practitioners are trained to take a generalist approach which allows them to deliver holistic, person-centred care. The wider multidisciplinary team is also important during the patient's HF journey with a particular need to consider palliative care input towards the end of life. This article summarises the important aspects of HF management in older people from the perspective of primary care.

Keywords: Heart failure; older people; primary care

\section{Introduction}

Heart Failure (HF) is a common condition that predominantly affects older people, where it is a leading cause of morbidity and mortality. A study of 156,013 patients with HF found the median age at diagnosis was 75 years [1]. The overall prevalence of HF in adults is $1-2 \%$ but $\geq 10 \%$ in those aged over 70 years old [2,3] but these figures may be a significant underestimate. Symptoms due to HF, such as breathlessness, often begin insidiously or can be confused with normal ageing, meaning patients may not initially present to medical services. Referral for further cardiological investigation may not be done immediately if other diagnostic possibilities, such as respiratory causes, are pursued first. Older people are therefore at higher risk of a late or missed HF diagnosis [4].

In contrast to younger populations where the underlying pathophysiology is often a single cause such as hypertrophic obstructive cardiomyopathy, HF in older people is usually related to a combination of underlying diseases such as hypertension or ischaemic heart disease $[5,6]$. This presents unique challenges including balancing treatment options in the context of polypharmacy and multi-morbidity. Older people with HF often require a generalist approach to management and most patients are managed by their general practitioner (GP) supported by the multidisciplinary HF team when necessary. This article provides a systematic evidence overview of primary care management of HF in older people.

\section{Methods}

A literature search was conducted on PubMed, EMBASE and the Cochrane library to identify relevant and recent material. The search focus was on systematic reviews and meta-analysis to provide the highest quality source material. Given the breadth of potentially pertinent information, we provide only a narrative overview of key data, focusing particularly on aspects of HF management that are most important or unique to older people. We also reviewed international guidelines to ensure the recommendations in the article align with accepted current best practice. 


\section{Background, categorisation \& prognosis}

$\mathrm{HF}$ is a clinical syndrome where reduced cardiac output due a 'structural or functional cardiac abnormality' results in typical symptoms (e.g. breathlessness, ankle swelling and fatigue) and signs (e.g. elevated jugular venous pressure, pulmonary crackles and peripheral oedema) [7]. Detecting and demonstrating the underlying cardiac abnormality is central to the diagnosis. The main historical categorisation of HF is based on the degree of impairment of the left ventricle, measured as the left ventricular ejection fraction (LVEF) and summarised in Table 1 [7]. These distinctions are important as the evidence base for treatment in HF with reduced ejection fraction (HFrEF) is well established, yet no treatment has as yet been proven to improve prognosis in HF with preserved ejection fraction (HFpEF).

At age 55 years, the lifetime risk of $\mathrm{HF}$ in men is 33\% and in women 28\% [8]. Prognosis depends on a number of factors including age at diagnosis, co-morbidities and LVEF. A 2017 survival analysis of 54,313 patients with a first diagnosis of HF in UK general practice between 1998 and 2012 found survival rates of $81.3 \%, 51.5 \%$ and $29.5 \%$ at 1,5 and 10 years respectively [9]. Despite the established evidence base for treatment in HFrEF, prognosis did not improve over the 15 year time period of this study. A Canadian database study comparing outcomes for 419,551 incident HF cases between 1997 and 2007 showed a reduction in 1 year mortality from $27.0 \%$ to $25.2 \%$ amongst all patients [10]. The increasing number of co-morbidities in older people, under treatment of HFrEF, and lack of effective treatment in HFpEF, which predominates in the elderly, are all potential reasons why a substantial improvement in outlook has not been seen in the community. Improved prognosis over time has been observed in patients admitted with acute HF [11].

\section{Pharmacological treatment options}

Figure 1 shows the European Society of Cardiology (ESC) flow diagram for treatment in HFrEF. International guidelines recommend that two main pharmacological therapies are used in all patients with HFrEF unless there is a contraindication; beta-blockers (BB) and angiotensin converting enzyme inhibitors (ACEI) or angiotensin receptor blockers (ARB) in those intolerant of ACEI [7, 12]. A third, mineralocorticoid receptor antagonists (MRA), should be added where patients with HFrEF remain symptomatic. Evidence from randomised controlled trials in the 1990s demonstrates these treatments can improve symptoms and prognosis [13-15]. Both BB and ACEI should be uptitrated until patients are on the maximum tolerated or recommended dose. [16].

If patients with HF remain symptomatic despite this triple combination of treatment additional therapies can be considered. Ivabradine has a rate limiting action without a negative inotropic effect and acts independently of BB or calcium channel antagonists by blocking the 'funny channel'. It is licensed where HF patients are in sinus rhythm with rate $>70$ beats per minute $(\mathrm{bpm})$ and should also be uptitrated aiming for heart rate $<65 \mathrm{bpm}$ [17]. Entresto is a combination of the neprilysin inhibitor, sacubitril and the ARB, valsartan and termed an angiotensin receptor neprilysin inhibitor (ARNI). It can be used in place of ACEI in those who have tolerated the ACEl but remain symptomatic. In the PARADIGM-HF trial, Entresto was compared to enalapril and was associated with a reduction in HF symptoms, hospital admissions and mortality [11]. Overall prognosis including cardiac death improved in the Entresto group, over and above the benefits of ACEI.

For those with resistant symptoms despite these treatment options there remain pharmacological options in digoxin or the combination of hydralazine / isosorbide dinitrate (H-ISDN). Digoxin can be used for rate control with improvement in morbidity but given the increasing evidence base for BB and ivabradine it is now third line. The risk of drug interaction, electrolyte disturbance and digoxin toxicity mean digoxin should be used with caution in older people. A recent meta-analysis including data from 326,426 participants treated with digoxin from 19 studies included 91,379 with HF and found digoxin use was associated with increased all-cause mortality in the HF group (hazard ratio 1.14, 95\% confidence interval 1.06-1.22) [18]. H-ISDN has been associated with survival benefit compared to placebo with greatest benefit in the elderly black subgroup [19]. It can be considered where ACEI or ARB are not tolerated or in addition to these treatments where patients remain symptomatic provided blood pressure is carefully monitored. However the three times a day dosing 
regime and common side-effects including headaches and dizziness can mean it is poorly tolerated. Diuretics are recommended in both HFrEF and HFpEF for symptom relief in patients with evidence of fluid overload.

As previously noted no treatment has been shown to be effective in improving survival times in HFpEF. Studies that have attempted to use treatments such as ACEI in older patients with HFpEF have shown a modest, non-significant trend towards reduced mortality and hospitalisation compared to placebo (HR 0.692: 95\% Cl 0.474-1.010; $P=0.055$ ) [20]. Co-morbidities such as AF or hypertension should be actively managed to improve symptom control and slow HFpEF progression.

\section{Acute and chronic HF}

Acute HF is defined by the ESC as a 'rapid onset or worsening of symptoms and/or signs of HF' where as chronic HF occurs in patients who have been diagnosed for 'some time' and are stable where symptoms are unchanged in the previous month [7]. There is a growing recognition that this delineation between acute and chronic HF is more likely to represent a spectrum of symptom stability on an underlying disease trajectory. Nonetheless, it can be helpful to consider disease stability at the time of assessment and consider why symptoms may have changed and how this may impact on treatment decisions.

Acute HF is the most common cause for hospital admissions in those aged $\geq 65$ years. Each year in the United States of America there are over 1 million hospitalisations for acute HF and 6 million inpatient days [21]. Numerous triggers may lead to a patient acutely decompensating, including infection, anaemia, non-adherence to treatment, new medication, acute coronary syndromes, surgery or exacerbation of co-existing respiratory disease such as chronic obstructive pulmonary disease (COPD), necessitating a thorough patient assessment to identify and treat reversible causes.

GPs must be able to identify acute HF or decompensation of chronic HF. Clinical assessment should pay particular attention to signs of congestion (breathlessness, lung crepitations, ankle swelling) and hypoperfusion (fatigue, cool peripheries) [7]. A patient centred approach is required to weigh the benefits of hospital admission against up-titration of treatment in the community in less severe cases and where local services are available. Hospital admission may provide timely investigations and more intensive treatment options but carries increased risks in the elderly, including falls, delirium, secondary infections and deconditioning [22].

In acute HF, diuretics are often either initiated or increased to reduce symptom burden with loop diuretics such as furosemide usually first line. Furosemide is usually used orally, but gut absorption is decreased in patients with significant fluid overload so intravenous administration may be more effective. Loop diuretics have a threshold dose below which they have no diuretic effect and this is dependent on renal function, meaning in patients with significant renal impairment doses below $80 \mathrm{mg}$ may have little effect [23]. Safety of loop diuretics must be considered particularly in the elderly as they cause electrolyte imbalances such as hyponatraemia, hypokalaemia and hypomagnaesemia with a link to cardiac arrhythmias.

The SOLVD trial found a 37\% increased risk of sudden cardiac death due to arrhythmias in patients treated with loop diuretics and this was replicated in a trial by the Digitalis Investigation Group (DIG) which found high doses of loop diuretics were associated with a 31\% increased risk of death $[24,25]$. These results are confounded by the possibility that those treated with higher diuretic doses were likely to be more unwell at the outset but do suggest caution and close monitoring are needed. In the elderly, diuretics have also been linked with delirium, urinary incontinence, orthostatic hypotension and increased risk of falls. Clinicians should therefore aim to use loop diuretics judiciously; the dose must be reviewed regularly and dose reduction considered once symptoms stabilised. Alternative treatment options such as thiazide diuretics or nitrates may be considered where there is a lack of response or contraindication to loop diuretics.

\section{Polypharmacy}


Patients with HF are almost inevitably on a complex combination of medication, with an average of 6.8 prescription medications per day excluding over the counter treatment [26]. In the elderly population with co-morbidities the average is 10 . Community or practice pharmacists can play an important role in identifying potential drug interactions and monitoring adherence and medication changes. The number of potential interactions and contraindicated medications preclude an individual assessment of each here but up to date online reference tools are available [26].The American Heart Association guidelines recommend regular medication reconciliation with pharmacist input where available, medication flow tools, cessation of nonessential medication, consideration of switching to combination medication where possible to reduce overall number of tablets and patient education on over the counter medication to avoid [26].

\section{Managing co-morbidities}

Frailty defines a syndrome of decreased resistance and reduced capacity to respond to environmental stress or illness. HF prevalence is strongly associated with frailty, with the Cardiovascular Health Study showing HF prevalence of $1.8 \%$ in the non-frail, compared to $14 \%$ in the frail, an adjusted odds ratio of 7.51 (95\% confidence interval 4.66-12.12) [27].

Cacciatore et al conducted a 12 year prospective cohort study which found chronic HF mortality increased progressively with a rise in Frailty Staging System (FSS) score; $45.5 \%$ of participants with chronic HF and a FSS of 1 were alive at 9 years compared to none of the participants who had chronic HF and a FSS $\geq 3$ surviving to that point [28]. A recent Australian study using primary care data found $99.1 \%$ of patients with HF had at least one co-morbidity and $53.4 \%$ had $\geq 6$ [29].

Cognitive impairment is common; a 2013 study of patients hospitalised with $\mathrm{HF}$ found $47 \%$ had some degree of cognitive impairment with mini-mental state score $<25 / 30$ [30]. Complex medication regimes can be a particular challenge for older people and may require additional input from primary care, nursing teams or use of appliances such as dossette boxes to improve compliance. The presence of comorbidities has frequently meant that complex elderly patients were excluded from clinical trials and so evidence is lacking on best treatment and instead guidelines extrapolate findings from younger populations to the older age groups.

\section{Non-pharmacological treatment options}

Physical activity is important to reduce the risk of deconditioning and has been shown to improve symptoms and exercise tolerance. Where available, stable patients should be considered for specialised cardiac rehabilitation, which has been shown to improve both symptom burden and prognosis in chronic HF [31]. These courses often have the added benefit of peer support networks and provide information on other lifestyle changes that can be done to reduce the risk of further cardiac damage. Even housebound patients can do chair-based cardiac rehabilitation and all patients should be encouraged to undertake consistent but achievable levels of physical activity.

There is limited evidence to support a low sodium diet, but avoiding high levels of dietary sodium is recommended along with low levels of saturated fats [32]. Overweight or obese patients should be encouraged to lose weight and this alone can help improve symptoms and exercise tolerance in some. Conversely, some older people with HF are underweight and require a thorough assessment to determine the underlying cause then appropriate management to achieve an optimal weight.

Smoking cessation support, appropriate vaccinations (e.g. influenza and pneumococcal) and patient education groups are also all important considerations and ideally should be embedded in primary care systems to ensure universal access to these services for all patients with HF.

\section{Device and interventional options}

Whilst decisions around device or surgical treatment in HF require a multidisciplinary approach in secondary care, it is useful for primary care physicians to be aware of these options and consider 
when referral may be indicated. Sudden cardiac death due to ventricular arrhythmias or bradycardia is common in HF and therefore implantable cardioverter-defibrillators (ICD) can reduce mortality in selected populations [7]. This includes survivors of previous cardiac arrest, those with documented ventricular arrhythmias and certain patients with symptomatic HF (NYHA II-III) despite optimal medical therapy for $>3$ months [7]. Patients with severe HF (NYHA IV), recent myocardial infarction or significant comorbidity are more likely to die from non-sudden cardiac death and so there is no decrease in mortality with ICD use in this group.

Cardiac resynchronisation therapy (CRT) with either defibrillator (CRT-D) or pacemaker (CRT-P) are recommended for patients with a LVEF $\leq 35 \%$ and either a prolonged QRS interval $\geq 150$ milliseconds or a QRS $\geq 120$ milliseconds with left bundle branch block [33]. Data from MADIT-CRT (Multicenter Automatic Defibrillator Implantation Trial With Cardiac Resynchronization Therapy) suggests there may be a limited therapeutic window in terms of inserting CRTs before HF is too advanced, with the best outcomes in terms of reduced mortality and hospitalisations plus improved reverse remodelling when LVEF is between 30-35\% [34].

Whilst access to echocardiograms may be limited in some areas to allow this degree of patient monitoring, annual ECG and review of symptom progression alongside knowledge of baseline echocardiogram results can be used in primary care to help identify potential candidates for CRT.

\section{The wider community team}

The complexity of HF management means patients will often benefit from a multidisciplinary team approach. Options include HF specialist nurses who can provide clinical assessments and medication reviews in community clinics, reducing the need for patients to visit hospital and providing a close link to specialist services in secondary care. Physiotherapists and occupational therapists may also be able to provide home cardiac rehabilitation or equipment input to improve quality of life and functional ability.

Telemonitoring can be used to capture biometric data such as oxygen saturations or blood pressure and potentially enable early identification and treatment of deteriorating patients. A 2015 systematic review showed telemonitoring could reduce mortality and hospital admissions in HF [35]. Pharmacists can review complex medication regimes and identify any significant interactions to improve patient safety. Ambulatory medical units may be able to provide near home or day case treatment such as furosemide or nitrate infusions to support patients remaining in their home environment.

Implementing such changes can be difficult at an individual patient level and may require system change within the local health service to facilitate improvements in care. Such services may also be costly.

\section{Palliative care}

GPs remain a key point of contact for patients with HF throughout their journey and may be asked to provide patients with information on prognosis, in particular to allow meaningful advanced care planning. Resuscitation should be discussed due to the risk of sudden cardiac death. Patients with ICDs should be aware of the implications of their device remaining activated if their prognosis is poor and have the option to deactivate the device if they do not want resuscitation. The risk of death from co-morbidities should also be discussed as patients more often die from a cause other than their HF.

Many patients with HF report that prognosis and disease progression are not discussed and some are therefore unaware of the implications of their diagnosis [36]. For others, the term 'heart failure' itself can have negative associations and lead to feelings of anxiety and depression. 
A holistic approach to HF is strongly recommended, considering the mental health of patients, their family and/or carers. Palliative care teams can be helpful in advising on symptom control and integrating the strands of physical and psychological care. Again this remains an underused resource, perhaps because it can be difficult to know when a patient with HF is deteriorating or for fear the referral may cause emotional distress [36].

\section{Conclusion}

Management of HF can be particularly challenging in older people but is an important area for education and resource investment given this is the population with the greatest disease prevalence. There are uncertainties around the applicability of the evidence-base for HF treatments in older people, particularly those who are frail, have several conditions and take many medications. A holistic generalist approach in primary care can allow all of the medical issues to be addressed, not just the HF, with a focus on the things that are most important to the patient. The wider primary care team, including physiotherapists, occupational therapists, palliative care services, along with HF specialist nurses and the secondary care team can work together to manage the needs of this important group of older people.

\section{Conflicts of interest: None}

Table 1 - European Society of Cardiology heart failure categories by ejection fraction [7]

\begin{tabular}{|l|l|l|l|}
\hline Category of heart failure & Abbreviation & $\begin{array}{l}\text { Left ventricular } \\
\text { ejection fraction }\end{array}$ & $\begin{array}{l}\text { Additional criteria to meet } \\
\text { diagnosis }\end{array}$ \\
\hline $\begin{array}{l}\text { Heart failure with reduced } \\
\text { ejection fraction }\end{array}$ & HFrEF & $<40 \%$ & Not required \\
\hline $\begin{array}{l}\text { Heart failure with mid-range } \\
\text { ejection fraction }\end{array}$ & HFmrEF & Ho-49\% & $\begin{array}{l}\text { Elevated levels of natriuretic } \\
\text { peptides and either relevant } \\
\text { structural heart disease or } \\
\text { diastolic dysfunction }\end{array}$ \\
\hline $\begin{array}{l}\text { Heart failure with preserved } \\
\text { ejection fraction }\end{array}$ & HFpEF & $\geq 50 \%$ & $\begin{array}{l}\text { Elevated levels of natriuretic } \\
\text { peptides and either relevant } \\
\text { structural heart disease or }\end{array}$ \\
\hline
\end{tabular}

Figure 1 - European Society of Cardiology guidelines for therapeutic management of symptomatic patient with heart failure and reduced ejection. Reproduced from 2016 ESC guidelines for the diagnosis and treatment of acute and chronic heart failure [7] 


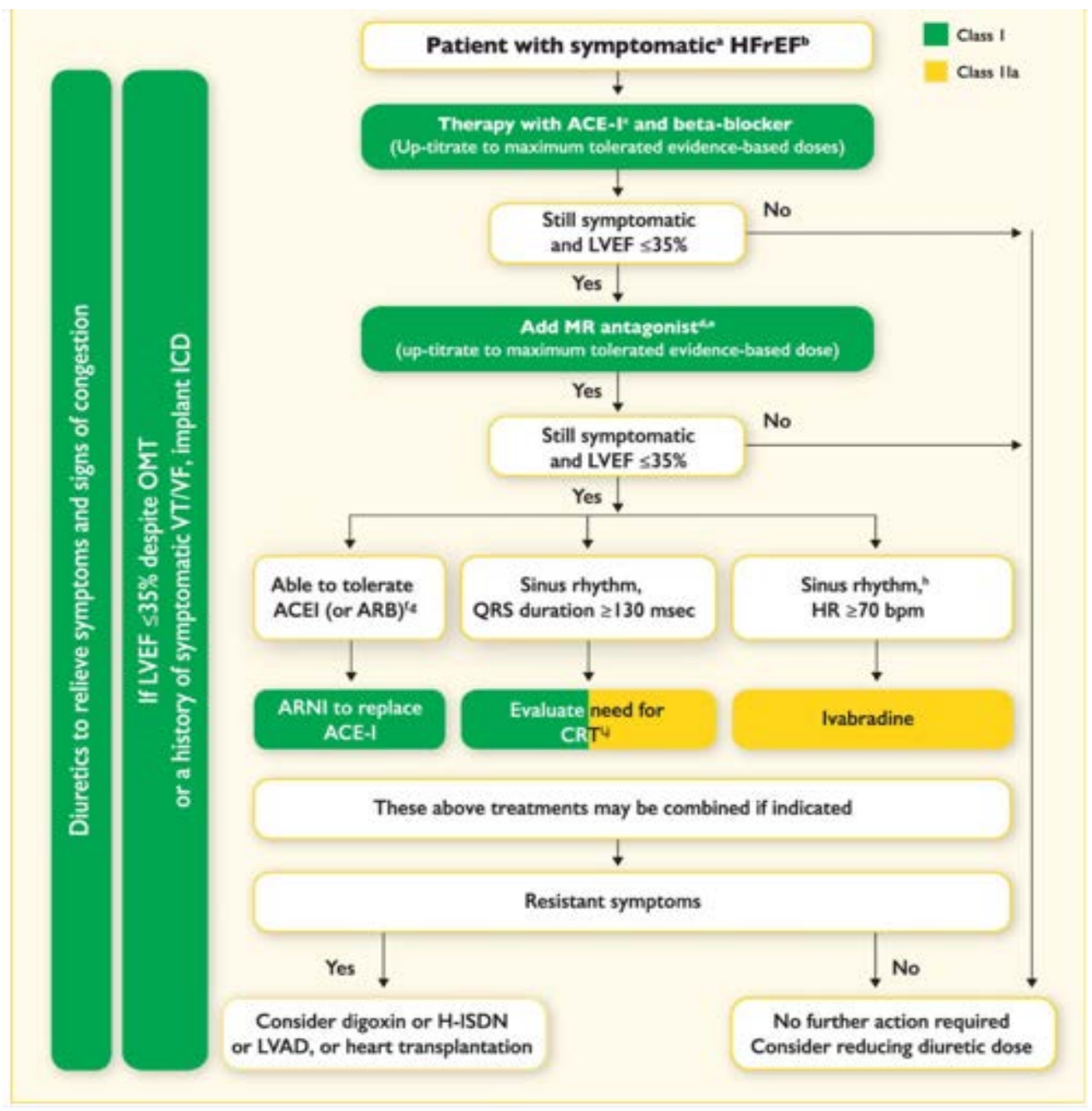

Green indicates a class I recommendation. Yellow indicates a class Ila recommendation.

Key - ACEI = angiotensin-converting enzyme inhibitor; $\mathrm{ARB}=$ angiotensin-receptor blocker; $\mathrm{ARNI}=$ angiotensin receptor neprilysin inhibitor; CRT = cardiac resynchronisation therapy; HFrEF = heart failure reduced ejection fraction; $\mathrm{H}-\mathrm{ISDN}=$ hydralazine and isosorbide dinitrate; HR = heart rate; ICD = implantable cardioverter defibrillator; LVAD = left ventricular assist device; LVEF = left ventricular ejection fraction; MR = mineralocorticoid antagonist; OMT = optimal medical therapy; VF = ventricular fibrillation; VT = ventricular tachycardia 


\section{References}

1. Shore, S., M. Grau-Sepulveda, D. Bhatt, H. PA, Z. Eapen, A. Hernandez, C. Yancy, and G. Fonarow, Characteristics, Treatments, and Outcomes of Hospitalized Heart Failure Patients Stratified by Etiologies of Cardiomyopathy. JACC Heart Fail., 2015 Nov. 3(11): p. 906-16.

2. Ceia, F., C. Fonseca, T. Mota, H. Morais, F. Matias, A. de Sousa, A. Oliveira, and EPICAInvestigators., Prevalence of chronic heart failure in Southwestern Europe: the EPICA study. Eur J Heart Fail., 2002 Aug. 4(4): p. 531-9.

3. Mosterd, A. and A. Hoes, Clinical epidemiology of heart failure. Heart, 2007 Sep. 93(9): p. 1137-1146.

4. Rutten, F., M. Cramer, D. Grobbee, A. Sachs, J. Kirkels, J. Lammers, and A. Hoes, Unrecognized heart failure in elderly patients with stable chronic obstructive pulmonary disease. Eur Heart J, 2005. 26(18): p. 1887-1894.

5. Maron, B., J. Gardin, J. Flack, S. Gidding, T. Kurosaki, and D. Bild, Prevalence of Hypertrophic Cardiomyopathy in a General Population of Young Adults: Echocardiographic Analysis of 4111 Subjects in the CARDIA Study. Circulation, 1995. 92(4): p. 785-789.

6. Metra, M., G. Cotter, J. El-Khorazaty, B. Davison, O. Milo, V. Carubelli, R. Bourge, J. Cleland, G. Jondeau, H. Krum, C. O'Connor, J. Parker, G. Torre-Amione, D. van Veldhuisen, M. Rainisio, I. Kobrin, J. McMurray, and J. Teerlink, Acute heart failure in the elderly: differences in clinical characteristics, outcomes, and prognostic factors in the VERITAS Study. J Card Fail, 2015 Mar. 21(3): p. 179-88.

7. 2016 ESC Guidelines for the diagnosis and treatment of acute and chronic heart failure: The Task Force for the diagnosis and treatment of acute and chronic heart failure of the European Society of Cardiology (ESC)Developed with the special contribution of the Heart Failure Association (HFA) of the ESC. Eur Heart J, 2016. 37(27): p. 2129-2200.

8. Bleumink, G., A. Knetsch, M. Sturkenboom, S. Straus, A. Hofman, J. Deckers, J. Witteman, and B. Stricker, Quantifying the heart failure epidemic: prevalence, incidence rate, lifetime risk and prognosis of heart failure. The Rotterdam Study. Eur Heart J, 2004. 25(18): p. 1614-1619.

9. Taylor, C., R. Ryan, L. Nichols, N. Gale, F. Hobbs, and T. Marshall, Survival following a diagnosis of heart failure in primary care. Fam Pract, 2017. 34(2): p. 161-168.

10. Yeung, F., N. Boom, H. Guo, D. Lee, S. Schultz, and J. Tu, Trends in the incidence and outcomes of heart failure in Ontario, Canada: 1997 to 2007. CMAJ, 2012. 184(14): p. E765E773.

11. McMurray, J., M. Packer, A. Desai, J. Gong, M. Lefkowitz, A. Rizkala, J. Rouleau, V. Shi, S. Solomon, K. Swedberg, and M. Zile, Angiotensin-Neprilysin Inhibition versus Enalapril in Heart Failure. From the PARADIGM-HF Investigators and Committees*. N Engl J Med, 2014. 371: p. 993-1004.

12. 2017 ACC/AHA/HFSA Focused Update of the 2013 ACCF/AHA Guideline for the Management of Heart Failure: A Report of the American College of Cardiology/American Heart Association Task Force on Clinical Practice Guidelines and the Heart Failure Society of America. Circulation, 2017.

13. Pitt, B., F. Zannad, W. Remme, R. Cody, A. Castaigne, A. Perez, J. Palensky, and J. Wittes, The effect of spironolactone on morbidity and mortality in patients with severe heart failure. Randomized Aldactone Evaluation Study Investigators. N Engl J Med. 341(10): p. 709-17.

14. Yusuf, S., B. Pitt, C. Davis, W. Hood, and J. Cohn, Effect of enalapril on survival in patients with reduced left ventricular ejection fractions and congestive heart failure. SOLVD Investigators. N Engl J Med, 1991. 325: p. 293-302.

15. The Cardiac Insufficiency Bisoprolol Study II (CIBIS-II): a randomised trial. CIBIS-II Investigators and Committees. Lancet, 1999. 353(9146): p. 9-13. 
16. Remme, W., G. Riegger, P. Hildebrandt, M. Komajda, W. Jaarsma, M. Bobbio, J. SolerSoler, A. Scherhag, B. Lutiger, and L. Rydén, The benefits of early combination treatment of carvedilol and an ACE-inhibitor in mild heart failure and left ventricular systolic dysfunction. The carvedilol and ACE-inhibitor remodelling mild heart failure evaluation trial (CARMEN). Cardiovasc Drugs Ther., 2004 Jan. 18(1): p. 57-66.

17. Swedberg, K., M. Komajda, M. Böhm, J. Borer, I. Ford, A. Dubost-Brama, G. Lerebours, i.L. Tavazz, and SHIFT-Investigators., Ivabradine and outcomes in chronic heart failure (SHIFT): a randomised placebo-controlled study. Lancet, 2010. 376(9744): p. 875-85.

18. Vamos, M., J. Erath, and S. Hohnloser, Digoxin-associated mortality: a systematic review and meta-analysis of the literature. Eur Heart J, 2015. 36(28): p. 1831-8.

19. Carson, P., S. Ziesche, G. Johnson, and J. Cohn, Racial differences in response to therapy for heart failure: analysis of the vasodilator-heart failure trials: Vasodilator-Heart Failure Trial Study Group. J Card Fail, 1999. 5(3): p. 178-187.

20. Cleland, J., M. Tendera, J. Adamus, N. Freemantle, L. Polonski, and J. Taylor, The perindopril in elderly people with chronic heart failure (PEP-CHF) study. Eur Heart J, 2006. 27(19): p. 2338-2345.

21. Joseph, S., A. Cedars, G. Ewald, E. Geltman, and D. Mann, Acute Decompensated Heart Failure: Contemporary Medical Management. Tex Heart Inst J, 2009. 36(6): p. 510-520.

22. Leape, L., T. Brennan, N. Laird, A. Lawthers, A. Localio, B. Barnes, L. Hebert, J. Newhouse, P. Weiler, and H. Hiatt, The Nature of Adverse Events in Hospitalized Patients — Results of the Harvard Medical Practice Study II. N Engl J Med, 1991. 324: p. 377-384.

23. Oh, S. and S. Han, Loop Diuretics in Clinical Practice. Electrolyte Blood Press., 2015. 13(1): p. 17-21.

24. Cooper, H., D. Dries, C. Davis, Y. Shen, and D. MJ, Diuretics and risk of arrhythmic death in patients with left ventricular dysfunction. Circulation, 1999. 100(12): p. 1311-5.

25. Ahmed, A., A. Husain, T. Love, G. Gambassi, L. Dell'Italia, G. Francis, M. Gheorghiade, R. Allman, S. Meleth, and R. Bourge, Heart failure, chronic diuretic use, and increase in mortality and hospitalization: an observational study using propensity score methods. Eur Heart J, 2006 Jun. 27(12): p. 1431-9.

26. Page, R., C. O’Bryant, D. Cheng, T. Dow, B. Ky, C. Stein, A. Spencer, R. Trupp, and J. Lindenfeld, Drugs That May Cause or Exacerbate Heart Failure: A Scientific Statement From the American Heart Association and On behalf of the American Heart Association Clinical Pharmacology and Heart Failure and Transplantation Committees of the Council on Clinical Cardiology; Council on Cardiovascular Surgery and Anesthesia; Council on Cardiovascular and Stroke Nursing; and Council on Quality of Care and Outcomes Research. Circulation, 2016. 134: p. e32-e69.

27. Newman, A., J. Gottdiener, M. Mcburnie, C. Hirsch, W. Kop, R. Tracy, J. Walston, and L. Fried, Associations of subclinical cardiovascular disease with frailty; Cardiovascular Health Study Research Group. J Gerontol A Biol Sci Med Sci, 2001 Mar. 56(3): p. M15866.

28. Cacciatore, F., P. Abete, F. Mazzella, L. Viati, D. Della Morte, D. D’Ambrosio, G. Gargiulo, G. Testa, D. De Santis, G. Galizia, N. Ferrara, and F. Rengo, Frailty predicts long-term mortality in elderly subjects with chronic heart failure. European Journal of Clinical Investigation, 2005. 35(12): p. 723-730.

29. Taylor, C., C. Harrison, H. Britt, G. Miller, and F. Hobbs, Heart failure and multimorbidity in Australian general practice. J of Comorbid, 2017. 7(1): p. 44-49.

30. Dodson, J., T. Truong, V. Towle, G. Kerins, and S. Chaudhry, Cognitive impairment in older adults with heart failure: prevalence, documentation, and impact on outcomes. Am J Med, 2013 Feb. 126(2): p. 120-6.

31. Ades, P., S. Keteyian, G. Balady, N. Houston-Miller, D. Kitzman, D. Mancini, and M. Rich, Cardiac Rehabilitation Exercise and Self-Care for Chronic Heart Failure. JACC: Heart Failure, 2013. 1(6): p. 540-547. 
32. Guidelines for the prevention, detection and management of chronic heart failure in Australia. National Heart Foundation of Australia. 2011.

33. NICE, Chronic heart failure in adults: management. Clinical guideline [CG108]. August 2010.

34. Moss, A., W. Hall, D. Cannom, H. Klein, M. Brown, J. Daubert, M. Estes, E. Foster, H. Greenberg, S. Higgins, M. Pfeffer, S. Solomon, and D. Wilber, Cardiac-Resynchronization Therapy for the Prevention of Heart-Failure Events. From the MADIT-CRT Trial Investigators. N Engl J Med, 2009. 361: p. 1329-1338.

35. Kitsiou, S., G. Paré, and M. Jaana, Effects of home telemonitoring interventions on patients with chronic heart failure: an overview of systematic reviews. J Med Internet Res, 2015. 17(3): p. e63.

36. Mcllvennan, C. and L. Allen, Palliative care in patients with heart failure. BMJ, 2016. 353: p. i1010. 